\title{
Impact of saturation on spin effects in proton-proton scattering*
}

\author{
O. V. Selyugin ${ }^{\dagger}$ and J.-R. Cudell ${ }^{\ddagger}$
}

November 24, 2018

\begin{abstract}
For pomerons described by a sum of two simple-pole terms, a soft and a hard pomeron, the unitarity bounds from saturation in impactparameter space are examined. We consider the effect of these bounds on observables linked with polarisation, such as the analyzing power $A_{N}$ in elastic proton-proton scattering, for LHC energies. We obtain the $s$ and $t$ dependence of the Coulomb-nuclear interference at small momentum transfer, and show that the effect of the hard pomeron may be observed at the LHC.
\end{abstract}

\section{Introduction}

The study of elastic scattering requires a detailed knowledge of the properties of the pomeron, which is the dominant interaction of hadrons at high energies. In this case, the structure and spin properties both of the hadron and of the pomeron play a special role.

There are two regimes for the pomeron, the "soft" non-perturbative pomeron, and the perturbative-QCD "hard" pomeron. The soft pomeron dominates in high-energy hadron-hadron diffractive reactions at long distances while the hard pomeron dominates in high-energy short-distance scattering [1] and determines the very-small- $x$ behaviour of deep inelastic structure functions and spin-averaged gluon distributions.

The "soft" pomeron of the standard form with $\alpha_{\text {pom }}(0)=1+\epsilon$. was introduced in 2] The observed growth of inelastic cross sections and the multiplicity coincide with these idea. The perturbative QCD leading log calculation of the gluon ladder diagrams gives the following result [3]

$$
\epsilon=12 \alpha_{s} / \pi \ln 2 \sim 0.5
$$

*presented by O.V.S. at the Advanced Studies Institute "Symetries and Spin" (SPINPraha-2004), Prague, July 5 - July 10, 2004.

$\dagger$ JINR, Bogoliubov Laboratory of Theoretical Physics, 141980 Dubna, Russia, e-mail: selugin@thsun1.jinr.ru

¥Institut de Physique, Bât. B5a, Université de Liège, Sart Tilman, B4000 Liège, Belgium, e-mail: JR.Cudell@ulg.ac.be 
Really, the new global QCD analysis of data for various hard scattering processes leads to the small $\mathrm{x}$ behaviour of the gluon structure function determined by the hard pomeron contribution [4 $g(x) \sim 1 / x^{1+\epsilon}$ with $\epsilon=0.3$. A recent analysis [5] of the experimental data for total cross sections and $\rho$-parameters of hadronhadron and photon-hadron scattering reveals the possibility that both soft and hard pomerons are simple poles, and that both contribute to soft physics: if the soft pomeron is a simple pole, the hard pomeron is in fact needed to describe simultaneously the energy dependence of the total cross sections (hence the imaginary part of the scattering amplitude at $t=0$ ) simultaneously with the value of $\rho(s, t=0)$ - the ratio of the real to the imaginary part of the hadron elastic spin-non-flip scattering amplitude - (hence the real part of this amplitude).

Note that the contribution of the hard pomeron to the elastic amplitude leads to the saturation of the unitarity bound in the profile function at some impact-parameter values at very high energies.

Here, by "saturation", we mean, in the $S$-matrix language, that for some distance between scattering particles, the maximum possible scattering is reached. This distance is correlated with the angular momentum $l$ and part of the scattering reaches the black disc limit (BDL).

It is that last meaning of "saturation", which is directly connected with the unitary property of the scattering amplitude, that we shall use in this work.

\section{The elastic-scattering amplitude at high en- ergy}

The presence of a hard pomeron in elastic diffractive processes with a large intercept $1+\epsilon_{2}=1.45[5$, 6] will lead to a lowering of the energy where saturation starts: it now appears as we approach the $\mathrm{TeV}$ region. It is not obvious how the total cross sections will grow with energy after partial saturation, especially in the energy region of LHC.

One can show that saturation leads to a behaviour of the total cross sections at LHC energy which is almost model-independent. Indeed, different models can be obtained by varying the the profile function of the hadrons. The BDL leads to a cut of this function when it reaches 1 . If we take a sharp cut with a break in the derivative, then we obtain strong edge effects from the disk corresponding to the radius of saturation $b_{s}$. To remove such nonphysical behaviour, we input an additional function which provides a smooth matching between the central black disk and the rest of the amplitude, and consider various profile functions (exponential, Gaussian, multipole).

We find in fact that the energy dependence of the imaginary part of the amplitude and hence of the total cross section depends on the form of $f(b)$, i.e. on the $s$ and $t$ dependence of the slope of the elastic scattering amplitude, but that fitting these quantities to existing data removes most of the uncertainty. It is unlikely that the LHC will help us choose the right profile function, or let us 
decide whether saturation has been reached. So, we need additional information.

Therefore, we investigate the impact of the hard pomeron on the polarisation of the elastic proton-proton scattering at LHC energies and at small momentum transfer. One of the most important effects is the Coulomb-Nuclear Interference at small momentum transfer [7, 8, 9, which mostly comes from the interference of the imaginary part of the hadron spin-non flip amplitude with the electromagnetic part of the spin-flip amplitude.

The differential cross section and analysing power $A_{N}$ are defined as follows:

$$
\begin{aligned}
\frac{d \sigma}{d t} & =\frac{2 \pi}{s^{2}}\left(\left|\Phi_{1}\right|^{2}+\left|\Phi_{2}\right|^{2}+\left|\Phi_{3}\right|^{2}+\left|\Phi_{4}\right|^{2}+4\left|\Phi_{5}\right|^{2}\right), \\
A_{N} \frac{d \sigma}{d t} & \left.=-\frac{4 \pi}{s^{2}} \operatorname{Im}\left[\left(\Phi_{1}+\Phi_{2}+\Phi_{3}-\Phi_{4}\right) \Phi_{5}^{*}\right)\right],
\end{aligned}
$$

in terms of the usual helicity amplitudes $\Phi_{i}$.

In the general case, the form of $A_{N}$ and the position of its maximum depend on the parameters of the elastic scattering amplitude: $\sigma_{\text {tot }}, \rho(s, t)$, the Coulomb-nuclear interference phase $\varphi_{\mathrm{cn}}(s, t)$ and the elastic slope $B(s, t)$. For the definition of new effects at small angles, and especially in the region of the diffraction minimum, one must know the effects of the Coulomb-nuclear interference with sufficiently high accuracy. The Coulomb-nuclear phase was calculated in the entire diffraction domain taking into account the form factors of the nucleons [10.

The total helicity amplitudes can be written as

$$
\Phi_{i}(s, t)=\phi_{i}^{h}(s, t)+\phi_{i}^{\mathrm{em}}(t) \exp \left[i \alpha_{\mathrm{em}} \varphi_{\mathrm{cn}}(s, t)\right],
$$

where $\alpha_{\mathrm{em}}=1 / 137$ is the electromagnetic constant, $\phi_{i}^{h}(s, t)$ describes the strong interaction of hadrons, $\phi_{i}^{\mathrm{em}}(t)$ their electromagnetic interaction, and $\varphi_{\mathrm{cn}}(s, t)$ is the electromagnetic-strong interference phase factor. Therefore, to determine the hadron spin-flip amplitude at small angles, one should take into account all electromagnetic and Coulomb-nuclear interference (CNI) effects.

In this paper, we define the hadronic and electromagnetic spin-non-flip amplitudes as

$$
\begin{aligned}
& F_{\mathrm{nf}}^{h}(s, t)=\frac{1}{2 s}\left[\phi_{1}^{h}(s, t)+\phi_{3}^{h}(s, t)\right] ; \\
& F_{\mathrm{nf}}^{c}(s, t)==\frac{1}{2 s}\left[\phi_{1}^{\mathrm{em}}(s, t)+\phi_{3}^{\mathrm{em}}(s, t)\right] .
\end{aligned}
$$

Taking into account the Coulomb-nuclear phase $\varphi_{\mathrm{cn}}$, we obtain

$$
\operatorname{Im} F_{\mathrm{nf}}^{c}=\alpha_{\mathrm{em}} \varphi_{\mathrm{cn}} F_{\mathrm{nf}}^{c} .
$$

Let us now examine the behaviour of the analysing power (3), which can be rewritten as

$$
\begin{aligned}
\frac{A_{N}}{2} \frac{d \sigma}{d t} & =\left(I m F_{\mathrm{nf}} R e F_{\mathrm{sf}}-R e F_{\mathrm{nf}} \operatorname{Im} F_{\mathrm{sf}}\right) \\
& =\left[\left(I m F_{\mathrm{nf}}^{h} R e F_{\mathrm{sf}}^{c}+I m F_{\mathrm{nf}}^{c} R e F_{\mathrm{sf}}^{c}-R e F_{\mathrm{nf}}^{h} \operatorname{Im} F_{\mathrm{sf}}^{c}-R e F_{\mathrm{nf}}^{c} I m F_{\mathrm{sf}}^{c}\right)\right. \\
& \left.+\left(I m F_{\mathrm{nf}}^{h} R e F_{\mathrm{sf}}^{h}-R e F_{\mathrm{nf}}^{c} \operatorname{Im} F_{\mathrm{sf}}^{h}+I m F_{\mathrm{nf}}^{c} \operatorname{Re} F_{\mathrm{sf}}^{h}-R e F_{\mathrm{nf}}^{h} I m F_{\mathrm{sf}}^{h}\right)\right]
\end{aligned}
$$


Equation (3) was applied at high energy and at small momentum transfer, with the following usual assumptions for hadron spin-flip amplitudes:

- $\phi_{1}=\phi_{3}, \phi_{2}=\phi_{4}=0$,

- the slopes of the hadronic spin-flip and spin-non-flip amplitudes are equal.

In this kinematic region, the analysing power can then be rewritten as

$$
\frac{A_{N}}{2} \frac{d \sigma}{d t}=-\left(I m F_{\mathrm{nf}}^{h} R e F_{\mathrm{sf}}^{c}+\operatorname{Im} F_{\mathrm{nf}}^{c} R e F_{\mathrm{sf}}^{c}\right)+\operatorname{Im} F_{\mathrm{sf}}^{c}\left(R e F_{\mathrm{nf}}^{c}+R e F_{\mathrm{nf}}^{h}\right)
$$

If we know the parameters of the hadron spin non-flip amplitude, the measurement of the analyzing power at small transfer momenta helps us to find the structure of the hadron spin-flip amplitude.

\subsection{Soft and hard pomeron as simple poles}

Let us extend the Donnachie-Landshoff (DL) 11] approach as it is a well-known model for hadron-hadron elastic scattering. A modern analysis of the existing experimental data for the hadron-hadron and photon-hadron total cross sections and $\rho(s, t=0)$ parameters suggests a possible contribution of the hard pomeron [5, 6].

In the DL approach, the $p p$-elastic scattering amplitude is proportional to the hadrons form-factors and can be approximated at small $t$ by [ $]$ :

$$
T(s, t)=\left[h_{1}\left(s / s_{0}\right)^{\epsilon_{1}} e^{\alpha_{1}^{\prime} t \ln \left(s / s_{0}\right)}+h_{2}\left(s / s_{0}\right)^{\epsilon_{2}} e^{\alpha_{2}^{\prime} t \ln \left(s / s_{0}\right)}\right] F^{2}(t) .
$$

where $h_{1}=4.47$ and $h_{2}=0.005$ are the coupling of the "soft" and "hard" pomerons, and $1+\epsilon_{1}=1.073, \alpha_{1}^{\prime}=0.3 \mathrm{GeV}^{2}$, and $1+\epsilon_{2}=1.45, \alpha_{2}^{\prime}=0.10 \mathrm{GeV}^{2}$ are the intercepts and the slopes of the two pomeron trajectories. The normalisation $s_{0}$ will be set to $1 \mathrm{GeV}^{2}$ below and $s$ implicitly contains the phase factor $\exp (-i \pi / 2) \cdot F^{2}(t)$ is the square of the Dirac elastic form factor,

$$
F^{2}(t)=\frac{4 m_{p}^{2}-2.79 t}{4 m_{p}^{2}-t} \frac{1}{1-t / \Lambda^{2}}
$$

where $m_{p}$ is the mass of the proton and $\Lambda^{2}=0.71 \mathrm{GeV}^{2}$. It can be approximated by the sum of three exponential [12]:

$$
F^{2}(t) \approx f_{a} e^{d_{a} t}+f_{b} e^{d_{b} t}+f_{c} e^{d_{c} t} .
$$

with

$$
f_{a}=0.55, \quad f_{b}=0.25, \quad f_{c}=0.20,
$$

and

$$
d_{a}=5.5 \mathrm{GeV}^{-2}, \quad d_{b}=4.1 \mathrm{GeV}^{-2}, \quad d_{c}=1.2 \mathrm{GeV}^{-2} .
$$

We then obtain in the impact parameter representation a specific form for the profile function $\Gamma(b, s)$ [13. One can see that at some energy and at small $b, \Gamma(b, s)$ reaches the black disk limit. For one-(soft+hard)-pomeron exchange 
this will be in the region $\sqrt{s} \approx 1.5 \mathrm{TeV}$. If one adds to the model the cut due to 2-pomeron exchanges [11, the resulting $\Gamma_{2}$ will saturate at $\sqrt{s}=4.5 \mathrm{TeV}$.

From the DL model, we obtain in the impact parameter representation the following form of the profile function $\Gamma(b, s)$ :

$$
\begin{aligned}
\Gamma(b, s) & =h_{1 a} \exp \left(-\frac{x^{2}}{r_{1 a}^{2}}\right)+h_{1 b} \exp \left(-\frac{x^{2}}{r_{1 b}^{2}}\right)+h_{1 c} \exp \left(-\frac{x^{2}}{r_{1 c}^{2}}\right) \\
& +h_{2 a} \exp \left(-\frac{x^{2}}{r_{2 a}^{2}}\right)+h_{2 b} \exp \left(-\frac{x^{2}}{r_{2 b}^{2}}\right)+h_{2 c} \exp \left(-\frac{x^{2}}{r_{2 c}^{2}}\right),
\end{aligned}
$$

where the variables $h_{i}, i=a, b, c$ are

$$
h_{1 i}=2 f_{i} h_{1} s^{\epsilon_{1}} / r_{1 i}^{2} ; \quad h_{2 i}=2 f_{i} h_{2} s^{\epsilon_{2}} / r_{2 i}^{2} .
$$

with

$$
r_{1 i}^{2}=4\left(d_{i}+\alpha_{1}^{\prime} \log \left(s / s_{0}\right)\right) ; \quad r_{2 i}^{2}=4\left(d_{i}+\alpha_{2}^{\prime} \log \left(s / s_{0}\right)\right) .
$$

Here the value $s$ contains the phase factor $\exp (-i \pi / 2)$. Saturation of the profile function will control the behaviour of $\sigma_{\text {tot }}$ at super-high energies. We implement saturation as explained above, by using a smooth matching between the saturated region $\left(\Gamma(b, s)=1, b<b_{s}\right)$ and the unsaturated one $(\Gamma(b, s)$ given by (13).

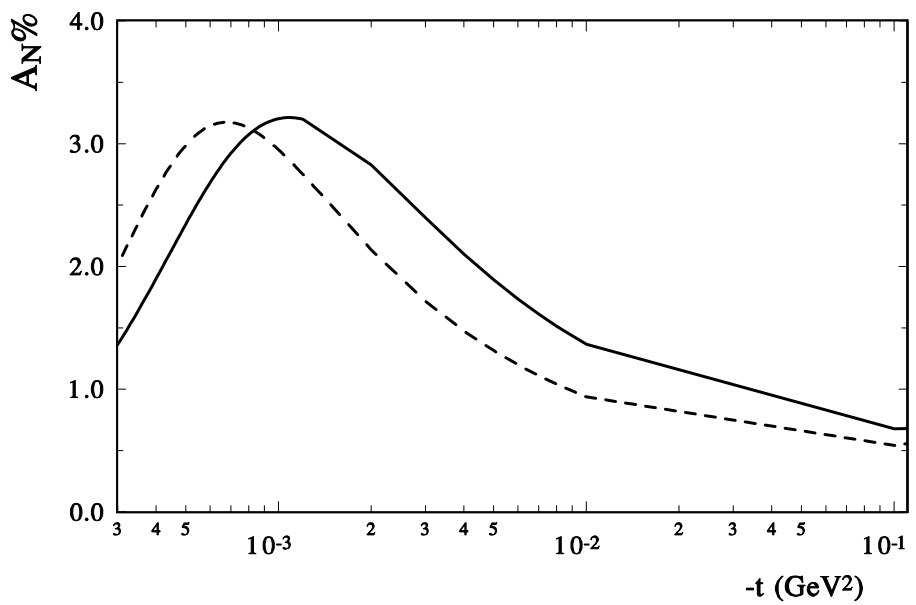

Figure 1: $A_{N}$ due to the interference of electromagnetic and strong amplitudes, calculated at $\sqrt{s}=20 \mathrm{TeV}$. (hard line - with saturation, with contributions from the soft and the hard pomerons; dashed line - without saturation, with a contribution only the soft pomeron, normalised to the total cross section at $\sqrt{s}=550 \mathrm{GeV})$.

Let us now calculate the CNI effect at LHC energies in different approaches. On the one hand, the contribution of the hard pomeron will lead to a growth of 
the real part of the spin-non-flip elastic scattering amplitude, but on the other hand, the contribution of the hard pomeron in the profile function at small impact parameters leads to the saturation of the unitarity bound, and to a decrease of the real part of the hadron spin-non-flip amplitude. The calculated $A_{N}$, which comes from the CNI-effect, in the framework of the DonnachieLandshoff model with a contribution from the hard pomeron is shown in Fig. 1.

In this figure, the hard line represents the result from saturation with contributions from the soft and from the hard pomerons whereas the dashed line is calculated without saturation but with only a soft pomeron. Of course the predictions for LHC energies depend on the values of the couplings and intercepts of the pomeron(s). For both cases, we choose these parameters to obtain a correct description of the total cross sections at high energies $(\sqrt{s}>50 \mathrm{GeV})$. From Fig. 1, we can see that the shape of the analysing power is practically the same in both cases.

In Fig. 2a, we show the energy dependence of the value of the maximum of $A_{N}$ for three cases. In the first one, we use saturation and contributions from the soft and the hard pomerons. In the second one, we do not use a unitarity/saturation bound and we allow a free growth of the profile function.

We then obtain a decrease of the maximum CNI-effect faster than in the case of the saturation regime but only at very high energies $(\sqrt{s}>10 \mathrm{TeV})$. This means that saturation tampers the growth of the real part which comes from the hard pomeron. Finally, the third case shows the standard behaviour of of the CNI effect when we take into account only the contribution of the soft pomeron (with intercept 1.07), which reaches the unitarity bound only at super-high energies beyond the LHC. At the LHC, the value of the maximum of $A_{N}$ changes slowly with the energy.

In Fig. 2b, we show the energy dependence of the value of the maximum of $A_{N}$ in these three cases. It increases faster in the case of the saturation regime especially at sufficiently large energies, whereas the smallest change corresponds to the standard analysis with a contribution from the soft pomeron only.

\section{Conclusion}

In the presence of a hard pomeron, saturation effects can change the behaviour of some characteristic features of the diffractive scattering amplitudes at the LHC. However, accurate measurements of the analysing power in the Coulomb-nuclear interference region can map the structure of the hadron spin-flip amplitude, and this will give us further information about the behaviour of hadronic interactions at large distances. Large-distance spin-flip contributions can be taken into account, for example in the peripheral dynamic model [14, 15. Saturation will lead to a relative growth of the contribution of peripheral interactions, and to changes in the energy dependence of the differential cross sections at moderate momentum transfer. This is especially true in the case of the energy dependence of the Coulomb-nuclear interference at small $t$. Such saturation effects can in principle be observed at the LHC. 

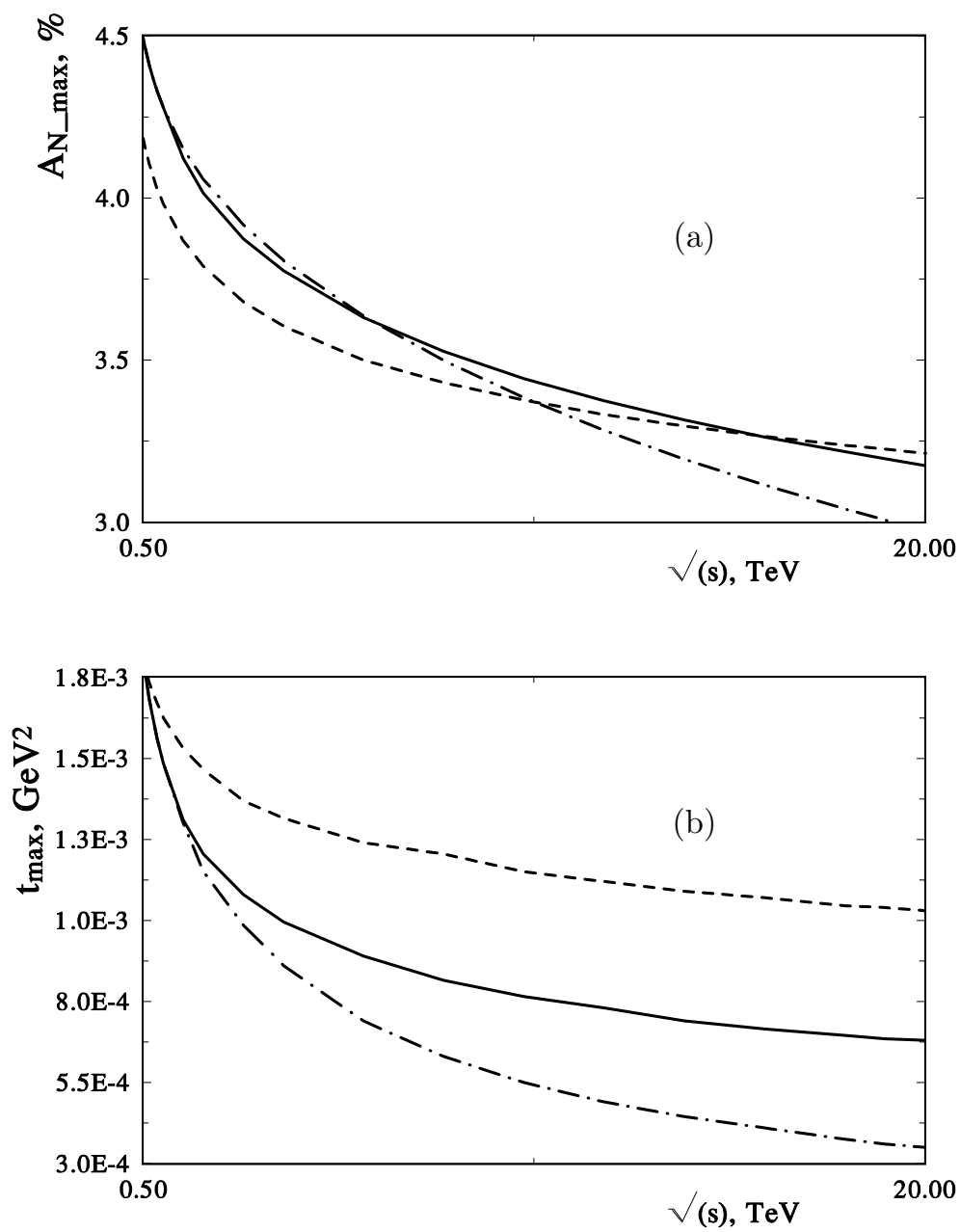

Figure 2: The energy dependence of a) the maximum value of $A_{N}$ and b) the position in $t$ of the maximum of $A_{N}$ (hard line: in the saturation regime, with contributions from the soft and the hard pomeron; the dash-dotted line: the same, but without saturation; dashed line: without saturation, with a contribution from the soft pomeron only, normalised to the total cross section at $\sqrt{s}=550 \mathrm{GeV})$. 
Acknowledgements: The authors would like to thank P.V. Landshoff for helpful discussion. This research was conducted while O.V.S. was a Visiting Fellow of the FNRS, Belgium.

\section{References}

[1] J. D. Bjorken: Nucl. Phys. Proc. Suppl. 25B (1992) 253.

[2] A.Donnachie, P.V.Landshoff, Nucl. Phys. v.231B (1984) 189.

[3] E.A.Kuraev, L.N.Lipatov, V.F.Fadin, Zetf, v.72 (1977) 377.

[4] H.L.Lai et al., preprint MSU-Hep-41024, STEQ-404, Michigan, 1994.

[5] J. R. Cudell, E. Martynov, O. Selyugin and A. Lengyel, Phys. Lett. B 587, 78 (2004) arXiv:hep-ph/0310198.

[6] A. Donnachie and P. V. Landshoff: Phys. Lett. B 595 (2004) 393, arXiv:hep-ph/0402081.

[7] J. S. Schwinger: Phys. Rev. 73 (1948) 416.

[8] L. I. Lapidus: Particles \& Nuclei 9 (1978) 84;

[9] N. H. Buttimore, E. Gotsman and E. Leader: Phys. Rev. D 18 (1978) 694;

[10] O. V. Selyugin: Phys. Rev. D 60 (1999) 074028.

[11] A. Donnachie and P. V. Landshoff: Nucl. Phys. B 231 (1984) 189.

[12] S. Donnachie, G. Dosch, O. Nachtmann and P. Landshoff: Pomeron Physics And QCD, Cambridge University Press (2002) p. 347, (Cambridge monographs on particle physics, nuclear physics and cosmology, 19).

[13] J.R. Cudell and O. V. Selyugin: in Proceedings of the 4th International Symposium on LHC Physics and Detectors (LHC 2003), Batavia, Illinois, May 1-3, 2003, arXiv:hep-ph/0309194.

[14] S. V. Goloskokov, S. P. Kuleshov and O. V. Selyugin, Z. Phys. C 50 (1991) 455.

[15] N. Akchurin, S. V. Goloskokov and O. V. Selyugin, Int. J. Mod. Phys. A 14 (1999) 253. 